Das gleiche gilt für die parallel als Opferschutz und präventiv wirkende Täterarbeit in Form von mit Tätern und Täterinnen häuslicher Gewalt durchzuführenden Verhaltenstrainings zur Verantwortungsübernahme. Diese bei Einführung des Gewaltschutzgesetzes nicht in den Vordergrund gestellte Arbeit muss nunmehr entsprechend der Zielsetzung im Aktionsplan II der Bundesregierung bundesweit vorangetrieben werden.

Der djb wird durch Mitwirkung in der B-L-AG Häusliche Gewalt und auf verschiedenen gesellschaftlichen Ebenen seinen Arbeitsanteil zur Umsetzung der Rechtspolitik für eine gewaltfreie Gesellschaft auch im sozialen Nahraum weiterhin erbringen. In der Verantwortung der Politik auf Bundes- und Landesebene wird es aber auch und gerade jetzt notwendig sein, neben der Beförderung der gesellschaftlichen Diskussion und konkreter Arbeitsschritte das für die Umsetzung erforderliche Geld in die Hand zu nehmen, damit langfristig die aus dem Bereich der häuslichen Gewalt verursachten enormen gesellschaftlichen Kosten gesenkt werden, die Erwachsenen, ihrer Vorbildfunktion entsprechend, von Religion und Herkunft unabhängig, ein gleichberechtigtes, gewaltfreies Zusammenleben vorleben, alle Frauen und Kinder in unserer Gesellschaft in einem gewaltfreien Umfeld friedlich leben und alle gemeinsam dieses Gesellschaftsmodell weitertransportieren können. Die engagierte bisherige Arbeit für diese Ziele hört nicht auf, sie hat erst angefangen und muss fortgeführt werden, ganz im Sinne des Zitats von Anne Frank: „Wie herrlich ist es, dass niemand auch nur eine Minute zu warten braucht, um damit zu beginnen, die Welt langsam zu verändern."

\title{
Häusliche Gewalt: Optimierungsmöglichkeiten im Bereich der Strafverfolgung
}

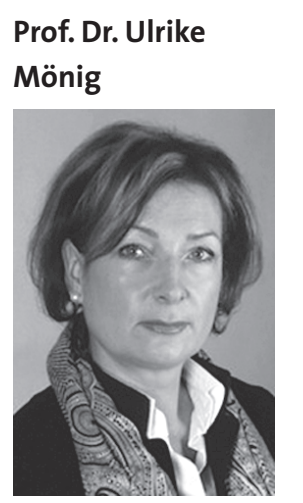

Mitglied der Kommission Gewalt gegen Frauen und Kinder des djb; Professorin an der Fachhochschule Bielefeld, Fachbereich Sozialwesen
Vor dem Hintergrund des angestrebten Paradigmenwechsels weg von der bagatellisierenden Behandlung häuslicher Gewalt und hin zu einer konsequenten Ächtung wurde im Wege einer Justizaktenanalyse die Erledigungspraxis der Strafjustiz nach Inkrafttreten des neuen Gewaltschutzrechts untersucht. ${ }^{1}$ Basierend auf den in der Studie gewonnenen Erkenntnissen werden im Folgenden einige Optimierungsmöglichkeiten zusammengefasst. ${ }^{2}$ Die Darstellung konzentriert sich auf Möglichkeiten im Bereich der Beweisführung (I), die Beurteilung des öffentlichen Interesses bei Verweisung auf den Privatklageweg (II) sowie die Betrachtung des Sanktions- und Verfahrensspektrums (III).

\section{Ausschöpfung aller Beweismöglichkeiten}

Auffällig ist, dass ein großer Teil der untersuchten Verfahren bereits im Ermittlungsverfahren durch die Staatsanwaltschaft gem. $\mathbb{1 7 0}$ Abs. 2 StPO eingestellt wurde. Von allen eingeleiteten Verfahren endeten 45,88 Prozent mit der Begründung mangelnden hinreichenden Tatverdachts. Die Diskrepanz zur entsprechenden Einstellungsquote bei allen Delikten (also z.B. auch Diebstahl), die im Vergleichszeitraum bundesweit 26,7 Prozent be$\operatorname{trug}^{3}$, ist erheblich - insbesondere, wenn man berücksichtigt, dass in dieser viel geringeren Einstellungsquote auch Verfahren gegen unbekannte Täter enthalten sind, während in Fällen häuslicher Gewalt die in Frage kommenden Personen mit allen Daten bekannt oder leicht ermittelbar sind. Fast die Hälfte der Einstellungen beruhte darauf, dass das Opfer als Angehöriger von dem ihm zustehenden Zeugnisverweigerungsrecht Gebrauch machte und deshalb als Beweismittel ausfiel. Dies muss jedoch nicht zwangsläufig zu einer Einstellung führen, wenngleich die Beweisführung dadurch ohne Zweifel schwieriger wird. Umso mehr muss sich das Augenmerk dann auf die Ausschöpfung der verbleibenden Beweismöglichkeiten richten. Da die Gründe für die fehlende Mitwirkung häufig im Fortwirken der Gewaltsituation liegen, wäre es ein fatales Signal, die fehlende Mitwirkung des Opfers grundsätzlich als fehlende Strafwürdigkeit zu interpretieren. $^{4}$

1 Die vollständigen Ergebnisse, die u.a. auch sozialstatistische Merkmale der Beteiligten und eine nähere Betrachtung der Taten und Tatumstände sowie des Aussageverhaltens der Beteiligten enthalten, wurden unter dem Titel „Häusliche Gewalt und die strafjustizielle Erledigungspraxis - Eine Justizaktenanalyse“ 2007 im Nomos Verlag in der Reihe „Mainzer Schriften zur Situation von Kriminalitätsopfern“ Bd. 43 veröffentlicht. Ausgewertet wurden insgesamt 247 Verfahren mit 255 Beschuldigten und 274 Opfern. Die Vorfälle ereigneten sich im ersten Halbjahr 2003 im Zuständigkeitsbereich des Polizeipräsidiums Bielefeld. Es wurde der Frage nachgegangen, auf welche Art und Weise bei der Polizei registrierte Vorgänge wegen häuslicher Gewalt im weiteren Verlauf des Strafverfahrens behandelt und abgeschlossen werden.

2 Teile des Textes sind der unter Fn. 1 genannten Veröffentlichung entnommen. Quellenangaben wurden aktualisiert.

3 Jehle, Strafrechtspflege in Deutschland, hrsg. vom Bundesministerium der Justiz, 4. Aufl., Berlin 2005, S. 20.

4 Vgl. Schirrmacher/Schweikert, Frauen, Männer und Gewalt - Neue Erkenntnisse zu Ausmaß, Folgen und wirksamen Interventionen, in: Familie, Partnerschaft, Recht 2005, S. 48. 


\section{Klärung der Aussagebereitschaft}

In der großen Mehrzahl aller Fälle, in denen sich die Einstellungsbegründung wesentlich auf die Ausübung des Zeugnisverweigerungsrechts durch die Opferzeugen stützte, war von diesem Recht ausdrücklich im Rahmen einer persönlichen Vernehmung, der die gesetzlich vorgeschriebene Belehrung vorausgegangen war, Gebrauch gemacht worden. Problematisch ist es jedoch, eine mangelnde Aussagebereitschaft auf Grund bestimmter, nicht immer eindeutiger Umstände lediglich zu unterstellen (Einstellungsverfügung der Staatsanwaltschaft: „Ehefrau macht offenbar von ibrem Zeugnisverweigerungsrecht Gebrauch ... "). ${ }^{5}$ Eine Ladung zur Vernehmung durch den Ermittlungsrichter mit dem Zusatz „Sofern Sie zum Termin nicht erscheinen, wird davon ausgegangen, dass Sie von Ihrem Aussageverweigerungsrecht Gebrauch machen wollen; die Angelegenheit hat sich dann erledigt. Sie müssen nicht mit Zwangsmaßnahmen (Ordnungsgeld oder Vorführung) rechnen. " lädt zur Untätigkeit geradezu ein. Die Wahl des rein passiven Verhaltens mag gerade für mit der Justiz nicht vertraute Opferzeugen in der konkreten Situation oft als der einfachere Weg erscheinen. Ferner ist zu berücksichtigen, dass ein Ausbleiben bei der Vernehmung nicht immer aus freien Stücken erfolgt, sondern auch darauf beruhen kann, dass die in deutscher Sprache verfasste Ladung nicht verstanden oder auf das Opfer Druck ausgeübt wird.

Auch die Aussagebereitschaft der beschuldigten Person wurde zuweilen nicht hinreichend geklärt. Festzustellen war, dass mehr als ein Viertel der Tatverdächtigen auf die Ladung durch die Polizei überhaupt nicht reagierten und Vernehmungen durch die Staatsanwaltschaft oder den Ermittlungsrichter nur äußerst selten durchgeführt wurden. So bleibt ein mögliches Beweisinstrument ungenutzt.

\section{Der Zeugenbeweis}

Zuweilen konnte festgestellt werden, dass eine konsequentere Nutzung des Zeugenbeweises zur Gewinnung (weiterer) sachdienlicher Erkenntnisse hätte beitragen können.

Nicht selten äußern sich Zeugnisverweigerungsberechtigte oder Beschuldigte unmittelbar nach dem Gewaltgeschehen spontan gegenüber einem Strafverfolgungsorgan, ohne dass eine Vernehmungssituation vorliegt. Dazu zählen zum Beispiel Angaben, die im Rahmen eines Notrufs oder unmittelbar nach Eintreffen der Polizei am Tatort ohne gezielte Befragung gemacht werden. Solche Spontanäußerungen sind nach herrschender Meinung uneingeschränkt verwertbar, auch wenn später vom Zeugnisverweigerungs- oder Aussageverweigerungsrecht Gebrauch gemacht wird. ${ }^{6}$ Die anwesenden Polizeibeamten können über den Inhalt dieser Angaben ebenso als Zeuge vernommen werden wie unbeteiligte Dritte. Hier empfiehlt sich eine möglichst genaue, im besten Fall wörtliche Dokumentation der Äußerungen.

Soweit es sich bei Zeugen um Kinder und Jugendliche handelte, wurde auf ihre Vernehmung manchmal bewusst verzichtet. Sicherlich sollte die Vernehmung von Kindern wegen der damit verbundenen Belastungen unterbleiben, wenn sie aus
Beweisgründen nicht notwendig erscheint. Problematisch ist es, wenn die Möglichkeit der Vernehmung kindlicher und jugendlicher Zeugen von vornherein nicht in Betracht gezogen wird. Auch Kinder unterliegen der allgemeinen Zeugnispflicht, sofern sie zeugnisfähig sind. Die gesetzlichen Vertreter haben das Kind auf Grund ihrer Erziehungspflicht zur Erfüllung seiner Zeugenpflicht anzuhalten. ${ }^{7}$ Zum Schutz kindlicher Zeugen existieren mittlerweile vielfältige Zeugenschutzmöglichkeiten während des gesamten Strafverfahrens. ${ }^{8}$ Kinder wurden zumeist sogar dann nicht vernommen, wenn sie selbst die Polizei gerufen haben. Es liegt auf der Hand, dass eine solche Vorgehensweise auch von den mitbetroffenen Kindern leicht als Bagatellisierung häuslicher Gewalt verstanden werden kann.

Nur ein recht kleiner Teil der Zeugen, die ohne nähere Angaben nicht bei der Polizei erschienen waren, wurde zur Vernehmung durch die Staatsanwaltschaft - dort besteht anders als bei der Polizei eine Erscheinenspflicht - geladen. 14,6 Prozent der Opferzeugen reagierten überhaupt nicht auf eine polizeiliche Ladung; lediglich 4,01 Prozent wurden durch die Staatsanwaltschaft geladen. Wird nicht ermittelt, was sie zum Tatgeschehen beitragen können, so kann dies leicht eine Einstellung mangels Tatverdachts begünstigen.

Auffällig ist die bei der Aktenauswertung festgestellte recht geringe Beteiligung der Opfer im Hauptverfahren. Die Praxis zeigt, dass in der Hauptverhandlung häufig auf Zeugenvernehmungen verzichtet wird, wenn ein Geständnis dem Grunde nach vorhanden ist. $\mathrm{Zu}$ bedenken ist jedoch, dass unter dem Druck der Verfahrensökonomie möglicherweise relevante Teilaspekte, die sich zu Ungunsten der angeklagten Person auswirken könnten, nicht weiter verfolgt werden. Im Opferinteresse liegt der Vernehmungsverzicht oft nicht, sondern er kommt in erster Linie der angeklagten Person zugute. Was aus Wirtschaftsstrafverfahren bekannt ist - Einräumen eines Teils der Vorwürfe unter Verzicht auf die Verfolgung weiterer sollte hier nicht Verbreitung finden, zumal es sich meistens um rechtlich und tatsächlich einfach gelagerte Fälle handelt.

\section{Fotografien als Augenscheinsobjekte}

Sehr hilfreich können Fotografien der Verletzungen und des Zustandes der Wohnung sein. Sie könnten als Augenscheinsobjekte Beweismittel im Prozess sein. Behauptungen, bestimmte Verletzungen oder Sachbeschädigungen hätten gar nicht vorgelegen, wären damit leicht zu entkräften. Angesichts der neueren leichten Fotoaufnahmetechniken könnte durchaus in einem größeren Umfang hiervon Gebrauch gemacht werden.

\section{Feststellung der Blutalkoholkonzentration}

Nicht selten versucht die beschuldigte Person, das schädigende Verhalten mit übermäßigem Alkoholkonsum, der zu verän-

\footnotetext{
5 Meyer-Goßner, Strafprozessordnung, 50. Aufl., München 2007. $\$ 52$ StPO Rn. 15, verlangt: „Ausdrücklich erklären muss der Zeuge seine Weigerung."

6 Beulke, Strafprozessrecht, 9. Aufl., Heidelberg 2006, Rn. 118.

7 Meyer-Goßner, $§ 48$ StPO Rn. 7.

8 Hierzu näher Laubenthal/Nevermann-Jaskolla, Die Rechte des Kindes als Zeuge im Strafverfahren, in: Juristische Arbeitsblätter 2005, S. 294 ff.
} 
derten Verhaltensweisen und Erinnerungslücken geführt habe, zu erklären. Eine damit verbundene Hoffnung auf milde strafrechtliche Bewertung wird oft genug mangels objektiver Beweise unter Anwendung des Grundsatzes „in dubio pro reo“ nicht enttäuscht. Die Rechtspraxis zeigt, dass in Fällen häuslicher Gewalt nur selten Blutuntersuchungen durchgeführt werden. Durch eine Steigerung der Blutprobenentnahmen würden stärker an objektiven Fakten orientierte Entscheidungen ermöglicht werden. ${ }^{9}$

\section{Prüfung des öffentlichen Interesses}

Bei Verweisungen auf den Privatklageweg kommt es auf das öffentliche Interesse an einer Verfolgung von Amts wegen an. Bei konsequenter Anwendung von Nr. 86 Abs. 2 der Richtlinien für das Straf- und Bußgeldverfahren dürfte dies im Regelfall zu bejahen sein. ${ }^{10}$ Obwohl der Anteil der nach $\mathbb{S} 376$ StPO eingestellten Verfahren mit 6,66 Prozent nicht hoch war, bedarf es stets genauer Prüfung, ob das öffentliche Interesse tatsächlich fehlt. $\mathrm{Zu}$ berücksichtigen ist insbesondere, dass die Begründung, es liege nur eine Familienstreitigkeit vor - womit zumeist argumentiert wird - durchweg nicht tragfähig ist. Gerade das Näheverhältnis wird in den Richtlinien als Grund für die Unzumutbarkeit der Privatklageerhebung gesehen. Auch wenn Beschuldigte und Geschädigte übereinstimmend vortragen, man habe sich ausgesöhnt und derzeit gebe es keine Probleme, ist dies unter Heranziehung der Erkenntnisse über Gewaltbeziehungen zu beurteilen. Nach Walker ${ }^{11}$ kann liebevolles Verhalten auch nur eine Phase in einer Gewaltbeziehung sein. Wenn Anhaltspunkte dafür vorliegen, dass die Beziehung seit längerem gewaltorientiert ist, kann die Verneinung des öffentlichen Interesses ein falsches Signal an die Beteiligten sein.

\section{Das Sanktions- und Verfahrensspektrum}

Bei der Betrachtung der verhängten Sanktionen - wobei der Begriff hier weit zu verstehen ist - und der gewählten Verfahren fällt auf, dass die Strafjustiz den Rahmen der eingeräumten Wahlmöglichkeiten nicht immer ausschöpft. Ein stärker an den individuellen Umständen des Falles ausgerichtetes und spezialpräventiv orientiertes Vorgehen könnte besser zur zukünftigen Vermeidung häuslicher Gewalt beitragen.

Dies gilt zunächst für Einstellungen nach $\mathbb{} 153 \mathrm{a}$ StPO, in dessen Rahmen hauptsächlich Geldauflagen oder gemeinnützige Arbeit verhängt wurden. Hier ist es inzwischen möglich, von den im Gesetz ausdrücklich vorgesehenen Möglichkeiten abzuweichen (,insbesondere“). ${ }^{12}$ Im Einzelfall kann sich zum Beispiel die Weisung, eine Paar- oder Suchtberatung aufzusuchen, anbieten oder es können Maßnahmen gewählt werden, die auch im Rahmen des Gewaltschutzgesetzes angeordnet werden könnten ${ }^{13}$, wobei auch dort der Katalog nicht abschließend ist. Zu berücksichtigen ist, dass eine Kombination verschiedener Weisungen im Rahmen des $\mathbb{1} 153$ a StPO durchaus möglich ist. ${ }^{14}$

Die untersuchten Verfahren wurden relativ häufig, nämlich in 10,71 Prozent aller im Vorverfahren beendeten Fälle, gem. \153b Abs. 1 StGB eingestellt, da die Voraussetzungen für das Vorliegen eines Täter-Opfer-Ausgleichs angenommen wurden. Bei dieser Vorgehensweise ist zu bedenken, dass sich gerade Reue und Einsicht einstellungsförderlich auswirken. Genau dieses Verhalten kann jedoch auch eine regelmäßig wiederkehrende Phase in einer gewaltorientierten Beziehung darstellen. ${ }^{15}$ Bei der Entscheidung ist daher besonderes Augenmerk auf die Vorgeschichte, insbesondere auf Wiederholungstaten, zu richten. Leicht könnte sonst eine staatliche Institution unfreiwillig zur Verfestigung bestehender Hierarchien in einer Gewaltbeziehung beitragen. Im Übrigen ist eine großzügige Anwendung des Täter-Opfer-Ausgleichs auf Fälle häuslicher Gewalt nicht unproblematisch, da die strafrechtliche Bewältigung von Gewaltgeschehen im sozialen Nahraum mit Hilfe von Gesprächen aushandelbar erscheint und damit bagatellisiert wird. ${ }^{16}$

Wird eine Freiheitsstrafe zur Bewährung ausgesprochen, so kann das Gericht bei der Festsetzung von Weisungen gem. \56c StGB wie bei $\mathbb{} 153$ a StPO von den ausdrücklich genannten Möglichkeiten abweichen und eine individuell passende Anordnung treffen. Wenngleich die Gerichte in den untersuchten Verfahren hiervon durchaus fallangemessen Gebrauch gemacht haben - zu erwähnen sind insbesondere Kontaktverbote, Aufnahme einer Suchttherapie und Aufnahme einer Psychotherapie -, sei auf diesen Spielraum noch einmal hingewiesen, da er erfahrungsgemäß nicht überall hinreichend genutzt wird. Bei Bewährungsauflagen gem. $\mathbb{S} 56 \mathrm{~b}$ StGB besteht dieser Spielraum zwar nicht; jedoch kann, wenn ein Geldbetrag zu zahlen ist, bei der Wahl einer gemeinnützigen Einrichtung in gewissem Umfang ein Fallbezug hergestellt werden. Sinnvoll in diesem Zusammenhang ist zum Beispiel die Zahlung an eine Einrichtung gegen Männergewalt oder an ein Frauenhaus.

9 Dabei ist der Verhältnismäßigkeitsgrundsatz zu beachten. Näher hierzu: Meyer-Goßner, $\S 81 a$ StPO Rn. 18.

10 Nach Schweikert/Baer, Das neue Gewaltschutzrecht, Baden-Baden 2002, Rn. 270, kann das öffentliche Interesse „nur ganz ausnahmsweise" nicht bejaht werden.

11 Walker, Warum schlägst du mich? Frauen werden misshandelt und wehren sich. Eine Psychologin berichtet, München 1994. Walker geht von insgesamt drei sich regelmäßig wiederholenden Phasen aus. In der englischen Originalausgabe (The battered woman, New York 1979) wird die Zyklustheorie der Gewalt („The Cycle Theory of Violence“) mit folgenden Phasen beschrieben: 1. „Tension-building Stage“, 2. , The Acute Battering Incident" und 3. ,Kindness and Contrite Loving Behavior" (S. 55 ff.). S. auch Godenzi, (Gewalt im sozialen Nahraum, 3. Aufl., Basel/Frankfurt a.M. 1996), der von 7 Phasen ausgeht; eine Kurzübersicht über diese Phasen befindet sich bei Schumacher/Janzen, Gewaltschutz in der Familie, Bielefeld 2003, Rn. 5.

12 Auf die verfassungsrechtlichen Grenzen weist Beulke, Rn. 337c, hin.

13 Näher hierzu Mönig, Die rechtlichen Schutzmöglichkeiten bei häuslicher Gewalt - Ein Beitrag zum neuen Gewaltschutzrecht, in: Zeitschrift des Interdisziplinären Frauenforschungs-Zentrums (IFFInfo) 2004, S. $8 \mathrm{ff}$.

14 Meyer-Goßner, § 153a StPO Rn.14.

15 Vgl. Fn. 11.

16 Kritisch zum TOA bei häuslicher Gewalt: Rabe, Der Täter-Opfer-Ausgleich bei häuslicher Gewalt, in: Streit 2002, S. 114 ff. Grundsätzlich kritisch zum TOA: Oberlies, Der Täter-Opfer-Ausgleich. Theorie und Praxis einer Glaubensrichtung, in: Streit 2000, S. 99 ff. 